\title{
Are ACOs Ready to be Accountable for Medication Use?
}

\author{
Robert W. Dubois, MD, PhD; Marv Feldman, RPh, MS; Adam Lustig, MS; Greg Kotzbauer: \\ Jerry Penso, MD, MBA; Scott D. Pope, PharmD; and Kimberly D. Westrich, MA
}

\begin{abstract}
BACKGROUND: Accountable care organizations (ACOs) have the potential to lower costs and improve quality through incentives and coordinated care. However, the design brings with it many new challenges. One such challenge is the optimal use of pharmaceuticals. Most ACOs have not yet focused on this integral facet of care, even though medications are a critical component to achieving the lower costs and improved quality that are anticipated with this new model.
\end{abstract}

OBJECTIVE: To evaluate whether ACOs are prepared to maximize the value of medications for achieving quality benchmarks and cost offsets.

METHODS: During the fall of 2012, an electronic readiness self-assessment was developed using a portion of the questions and question methodology from the National Survey of Accountable Care Organizations, along with original questions developed by the authors. The assessment was tested and subsequently revised based on feedback from pilot testing with 5 ACO representatives. The revised assessment was distributed via e-mail to a convenience sample $(n=175)$ of ACO members of the American Medical Group Association, Brookings-Dartmouth ACO Learning Network, and Premier Healthcare Alliance.

RESULTS: The self-assessment was completed by 46 ACO representatives (26\% response rate). ACOs reported high readiness to manage medications in a few areas, such as transmitting prescriptions electronically $(70 \%)$, being able to integrate medical and pharmacy data into a single database $(54 \%)$, and having a formulary in place that encourages generic use when appropriate $(50 \%)$. However, many areas have substantial room for improvement with few ACOs reporting high readiness. Some notable areas include being able to quantify the cost offsets and hence demonstrate the value of appropriate medication use $(7 \%)$, notifying a physician when a prescription has been filled ( $9 \%)$, having protocols in place to avoid medication duplication and polypharmacy (17\%), and having quality metrics in place for a broad diversity of conditions (22\%).

CONCLUSIONS: Developing the capabilities to support, monitor, and ensure appropriate medication use will be critical to achieve optimal patient outcomes and ACO success. The ACOs surveyed have embarked upon an important journey towards this goal, but critical gaps remain before they can become fully accountable. While many of these organizations have begun adopting health information technologies that allow them to maximize the value of medications for achieving quality outcomes and cost offsets, a significant lag was identified in their inability to use these technologies to their full capacities. In order to provide further guidance, the authors have begun documenting case studies for public release that would provide ACOs with examples of how certain medication issues have been addressed by ACOs or relevant organizations. The authors hope that these case studies will help ACOs optimize the value of pharmaceuticals and achieve the "triple aim" of improving care, health, and cost.

J Manag Care Pharm. 2014;20(1):17-21

Copyright $\odot 2014$, Academy of Managed Care Pharmacy. All rights reserved.

\section{What is already known about this subject}

- Optimizing medication use can result in cost and quality improvements.

- The Congressional Budget Office will include a medication use "offsetting" effect on spending for Medicare medical services.

- A previously published framework has explored the role of medication in achieving accountable care organization (ACO) cost and quality benchmarks.

\section{What this study adds}

- An overview of a survey tool designed to evaluate how ready ACOs are to optimize the value of pharmaceuticals.

- A presentation of the readiness survey results for 46 ACOs.

- An analysis of where these ACOs are succeeding or falling short in implementing the processes needed.

A ccountable care organizations (ACOs) are gaining traction among both private and public payers. The Department of Health and Human Services (HHS) recently named 254 ACOs under the Medicare Shared Savings and Pioneer programs, providing care to approximately 4 million Medicare beneficiaries. ${ }^{1}$ With more than 150 private ACOs existing prior to the most recent announcement of HHS, ${ }^{2} 1$ out of every 10 patients in the United States now receives care through an ACO. ${ }^{3}$ These developing provider organizations, ranging from new organizations or combinations of organizations to existing organizations with increased accountability, have the potential to lower costs and improve quality through coordinated care, with incentives based upon shared savings and achieving specified clinical benchmarks. However, the design brings with it many new challenges. One such challenge is the optimal use of pharmaceuticals. Most ACOs have not yet focused on this integral facet of care, even though medications are a critical component to achieving the lower costs and improved quality that are anticipated with this new model. While there are critics of the innovative aspects of ACOs, ${ }^{4}$ this model of care is continuing to evolve as lessons are learned and health systems develop new partnerships to account for all facets of care, including medications. This change was no more apparent than in the latest round of ACO announcements made by the HHS that included Walgreens, the first significant pharmacy partnership for an ACO. ${ }^{5}$ By partnering with 3 large physician groups in various parts of the country, Walgreens 
is acknowledging the critical role pharmacists play not only behind the counter in their more traditional role, but as an integral partner in patient care. With retail pharmacies now providing basic health services in their stores, such as vaccinations and preventive check-ups, these types of partnerships have the potential to improve patient care as ACOs continue to form and evolve.

The importance of medications is reflected in the Medicare Shared Savings quality measures where more than half will be impacted by optimal medication use, either directly (e.g., receiving beta-blocker therapy for left ventricular systolic dysfunction) or indirectly (e.g., blood pressure control). Optimizing medication use can improve patient outcomes. In certain circumstances, it can also reduce costs. As an example, research has shown that interventions to optimize heart failure medications reduced hospital readmission rates. ${ }^{6}$ This reduction saves money and also contributes to an important quality metric. In July 2012, the authors, in collaboration with 7 distinguished provider organizations, published a framework exploring the role of medications in helping an ACO lower cost growth and achieve desired quality benchmarks. The framework emphasizes 4 considerations: (1) understanding the impact of medications upon the full episode cost; (2) proactive consideration of medications as an essential element of full comprehensive condition management; (3) using composite risk to identify patients who are candidates for enhanced medication management support; and (4) implementing quality metrics to detect underuse when there are condition-specific incentives to encourage economic savings. ${ }^{7}$ Moving from theoretical framework to action, we designed and fielded a pharmaceutical readiness survey to assess ACO capabilities to implement these concepts.

\section{Methods}

A 28-item self-assessment was designed to evaluate whether ACOs are prepared to maximize the value of medications for achieving quality benchmarks and cost offsets. A portion of the questions and question methodology were selected from the National Survey of Accountable Care Organizations. Additional questions were drafted by the study authors. The assessment was fielded and subsequently revised based on feedback from pilot testing from 5 ACOs.

This cross-sectional readiness survey recruited a convenience sample of ACOs $(n=175)$ between August 2012 and November 2012 from members of the American Medical Group Association, Brookings-Dartmouth ACO Learning Network, and Premier Healthcare Alliance. These organizations' members represent a wide variety of ACO configurations, including group practices, independent practice associations, academic health systems, and integrated delivery systems.

The survey included 3 questions pertaining to the respondents' demographic information (role, background, length of employment) and 7 questions regarding organizational demographics (health plan ownership, type of provider system, number of hospitals/physicians, inclusion of long-term care/specialty offices, number of lives covered in largest ACO contract, and type of contract). For the 17 questions addressing ACOs' pharmaceutical readiness, a behavioral anchoring scale used questions with a response range of 1-9, with 1 being the least ready and 9 being the most ready. For each question, 3 anchors represented low readiness (a response of 1-3), moderate readiness (a response of 4-6), and high readiness (a response of 7-9). Higher numbers represent greater organizational readiness to address that issue. These questions addressed formulary processes, data systems, communication/ reporting capabilities, care plan management strategies, and contract structure. For example, for the question "To what extent do physicians across the ACO have the ability to transmit prescriptions electronically to pharmacies via a computer or mobile device?" response options were as follows:

- None. This functionality is not currently available. (1-3, low level of readiness)

- A portion of our physicians have this capability, and we are working to deploy the functionality across the system. (4-6, moderate level of readiness)

- All of our physicians have access to this functionality, and we are actively monitoring usage and the impact on system performance. (7-9, high level of readiness)

\section{Results}

\section{Individual and Organizational Characteristics}

The survey was completed by $26 \%$ of all contacted individuals $(\mathrm{N}=46)$, all of which self-identified their organizations as an ACO. Exactly $50 \%$ of the respondents have a medical role (e.g., Chief Medical Officer or Medical Director) and the other half have a pharmaceutical role (e.g., Chief Pharmacy Officer or Pharmacy Director). The most prevalent type of provider system was an integrated delivery system, which made up 37\% of all responses $(n=17)$, followed by physician practice-based ACOs $(30 \%, n=14)$, and hospital-based systems $(20 \%, n=9)$. Most of the respondents were part of medium-to-large health systems, with $28 \%(n=13)$ indicating their health systems employed 100-300 physicians, 33\% ( $n=15)$ employed 300 to 1,000 physicians, and $24 \%(n=11)$ employed more than 1,000 physicians. Approximately 15\% ( $\mathrm{n}=7)$ of all respondents indicated their health systems employed 100 physicians or fewer. Further reflecting the size of the ACOs surveyed, 37\% ( $\mathrm{n}=17)$ of all respondents indicated that they have 2 to 5 hospitals in their systems, with an additional 30\% ( $n=14)$ stating their systems included 5 or more hospitals.

There were significant differences in the number of lives covered by the respondents' largest ACO contracts. Approximately $15 \%(n=7)$ of the respondents indicated their largest contract covers less than 10,000 lives, $33 \%(n=15)$ cover 10,000 to 20,000 


\section{FIGURE 1 What Are ACOs Doing? Percentage of ACOs Surveyed That Reported High Readiness ${ }^{\mathrm{a}}$}

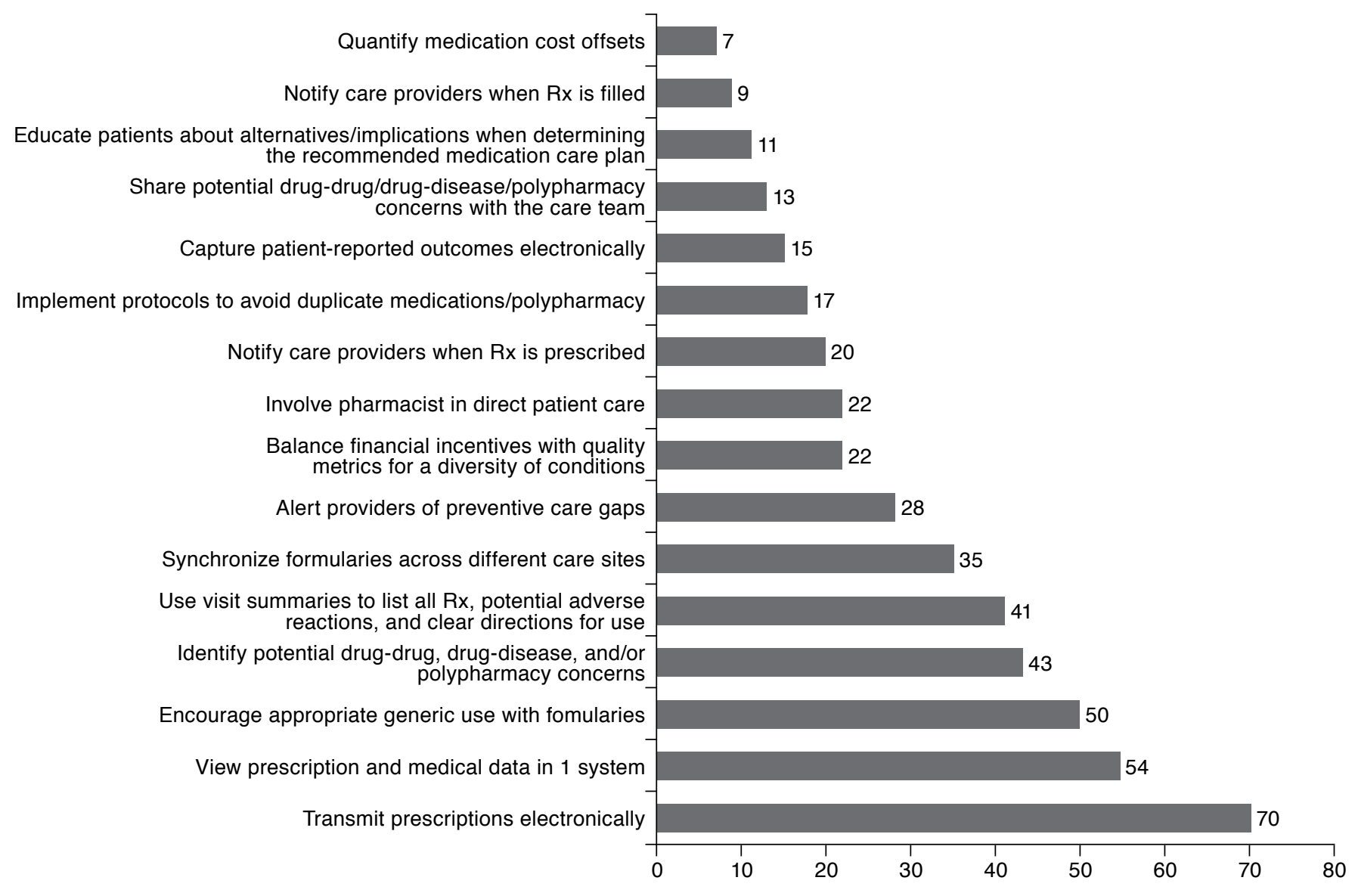

a Figure lists the topics from the survey and indicates the percentage of survey respondents who reported a high degree of readiness for each topic. Respondents were given a behavioral anchoring scale with response choices from 1 to 9. Choosing 1 to 3 indicated a low level of readiness, 4 to 6 a moderate level of readiness, and 7 to 9 a high level of readiness.

$R x=$ prescription.

lives, 30\% ( $n=14)$ cover 20,000 to 50,000 lives, and 22\% ( $n=10)$ cover more than 50,000 lives. Of these contracts, $41 \%(n=19)$ are with a Centers for Medicare and Medicaid Services (CMS) ACO program (Physician Group Practice Transition, Pioneer ACO, Medicare Shared Savings Program, or Comprehensive Primary Care Initiative); $24 \%(n=11)$ have agreements with regional commercial insurers; and 9\% $(n=4)$ have agreements with national commercial insurers. Other contract partners identified (26\%, $\mathrm{n}=12$ ) include the Department of Defense and Medicaid.

\section{Pharmaceutical Readiness}

We found that ACOs are ready to manage medications in a few selected areas (Figure 1). Seventy percent of respondents $(n=32)$ reported high readiness in the ability to transmit prescriptions electronically. Only 2 other topics had $50 \%$ or more of the ACOs reporting high readiness-the ability to integrate medical and pharmacy data into a single database $(54 \%, n=25)$ and having a formulary in place that encourages generic use when appropriate $(50 \%, \mathrm{n}=23)$.

There are safety-related topics as well. While $41 \%$ of the ACOs' visit summaries listed all drugs, potential adverse events, and clear directions for use $(n=19)$, with an additional $43 \%$ reporting the ability to identify potential drug-drug, drug-disease, and polypharmacy concerns $(n=20)$, only $13 \%$ of those surveyed have protocols in place to share identified adverse events and potentially dangerous interactions to patients' care teams when they occur $(n=6)$, and only $17 \%$ have protocols in place to avoid these types of issues $(n=8)$.

The readiness assessment also identified substantial room for improvement for provider and patient engagement efforts. Only $28 \%$ of the ACOs surveyed have the ability to alert physicians of gaps in preventive care $(n=13) ; 20 \%$ have the 
ability to notify a physician when a medication is prescribed by someone else on a patient's care team $(n=9)$; and only $9 \%$ have the ability to notify a physician when a prescription is filled $(n=4)$. In addition, $22 \%$ of the ACOs reported that pharmacists are involved in direct patient care $(n=10)$. While patient registries have been heralded as an effective way to monitor patient outcomes, only $22 \%$ of the ACOs surveyed have registries that track patients and medication use $(n=10)$. Furthermore, only $15 \%$ of those surveyed reported that their electronic medical record (EMR) system includes patient-reported outcomes $(n=7)$. Reflecting a lack of shared decision making, only $11 \%$ of the ACOs reported that patients are formally educated about therapeutic alternatives $(n=5)$.

\section{Discussion}

The data from this readiness assessment indicate that for most of the surveyed ACOs significant improvements are needed if these organizations are to optimize medication use and improve patient outcomes. The organizations surveyed demonstrate technological infrastructure capabilities, with $70 \%$ able to transmit prescriptions electronically and 50\% able to integrate medical and pharmacy data into a single database. These more positive findings may reflect organizations' responses to the CMS Meaningful Use incentive program. Previous research has shown that EMR adoption by hospitals rose by $75 \%$ between 2008 and 2011, ${ }^{8}$ and given that e-prescribing and integrating pharmacy data into a single database are key features of an EMR system, these results are not surprising. Further highlighting cost constraints on ACOs, 50\% of the ACOs surveyed encourage generic medications when appropriate. These data reflect previous research that demonstrated generic substitution decision support for e-prescribing significantly increases the proportion of generic drugs prescribed ${ }^{9}$ and lowers costs. ${ }^{10}$

Although ACOs are addressing certain medication management issues well, a significant area for improvement is how they address and communicate adverse events. A troubling finding is the lack of protocols in place to communicate (13\%) and manage (17\%) adverse events. Of note, few ACOs had a clinical pharmacist integrated into care teams. Without medication adverse-event protocols or clinical pharmacist integration into care teams, ACOs may lack the ability to ensure appropriate medication use and safety. A previous meta-analysis that included 298 studies found that integrating pharmacists in a patient's care team significantly improved adverse drug events $(P<0.05) .{ }^{11}$ Given that the Institute of Medicine cited preventable adverse events as a leading cause of death in the United States, ${ }^{12}$ the development of protocols to communicate and manage adverse events should be an ACO priority. With the federal Hospital Readmissions Reduction Program reducing readmission payments for select conditions on the fee-forservice side, ${ }^{13}$ and with ACOs taking on more financial risk via bundled payments and pay-for-performance contracts, ACOs will need to adopt and implement protocols to reduce these avoidable events.

With a growing emphasis being placed on shared decision making, ${ }^{14}$ and patient engagement being measured as a critical component of ACOs' quality metrics, ${ }^{15}$ ACOs will need an information technology infrastructure that allows for providers to effectively and efficiently communicate with patients. The ACOs in this assessment have room for improvement with respect to patient engagement efforts, considering the following: (a) a lack of patient-reported outcome integration in EMRs (15\%); (b) education about therapeutic alternatives (11\%); (c) use of patient registries (22\%); (d) a lack of ability by physicians to identify when a medication is prescribed (20\%) and filled (9\%), although neither reflects actual medication use; and (e) not being able to identify gaps in preventive care (28\%). Improving readiness in these areas will greatly facilitate ACO achievement of overall economic and quality performance goals.

The need for more sophisticated quality measures in order to adequately evaluate the impact of medication use was also highlighted by the assessment data. The CMS Shared Savings Program has metrics to evaluate clinical outcomes for conditions such as heart failure and diabetes. Similar quality metrics are not in place for conditions that often require more costly specialty drug use (e.g., rheumatoid arthritis, multiple sclerosis, cancer, hepatitis C). In these circumstances, ACOs may have incentives to pursue cost reductions without corresponding quality indicators to ensure optimal care. Only 22\% of the ACOs surveyed reported high readiness to use quality metrics in these situations. ACOs are rightfully cautious about facing an overwhelming number of quality metrics. Yet, it is important to have metrics in place to demonstrate that optimal care has been provided for both important prevalent and less prevalent conditions.

\section{Limitations}

A few important limitations of this study should be noted. The ACOs who participated in the assessment were a convenience sample of members from the American Medical Group Association, the Brookings-Dartmouth Learning Network, and Premier Healthcare Alliance. The data presented in this article may not be representative of all ACOs, since every health system is unique and has its own set of characteristics and patient populations. We did have a blend of ACO types and sizes and an equal mix of those with a pharmacy or medical role. Given the low rate of readiness, the sampling biases would have to be substantial to greatly impact the key study findings.

\section{Conclusions}

Developing the capabilities to support, monitor, and ensure appropriate medication use will be critical to optimal patient outcomes and ACO success. The ACOs in this assessment have 
embarked upon an important journey to achieve this goal, but critical gaps remain before they can become fully accountable. As a starting point, ACOs could use the authors' questionnaire (see Appendix, available in online article) to assess areas of current readiness and weakness and focus attention upon the latter. In order to provide further guidance, the authors have begun documenting case studies for public release that would provide ACOs with examples of how certain medication issues have been addressed by other organizations. The authors hope that these case studies will help ACOs improve their future consideration of pharmaceuticals and achieve the "triple aim" of improving care, health, and cost. ${ }^{16}$

\section{Authors}

ROBERT W. DUBOIS, MD, PhD, is Chief Science Officer; ADAM LUSTIG, MS, is Research Associate; and KIMBERLY D. WESTRICH, MA, is Director, Health Services Research, National Pharmaceutical Council, Washington, DC. MARV FELDMAN, $\mathrm{RPh}, \mathrm{MS}$, is Senior Director, Medication Management, and Managing Principal, Pharmacy Consulting, and SCOTT D. POPE, PharmD, is Executive Director, Healthcare Innovators Collaborative, Premier Healthcare Alliance, Charlotte, North Carolina. GREG KOTZBAUER is Project Manager, Health Policy \& ACO

Demonstrations, The Dartmouth Institute, Hanover, New Hampshire, and JERRY PENSO, MD, MBA, is Chief Medical and Quality Officer, American Medical Group Association, Washington, DC.

AUTHOR CORRESPONDENCE: Robert W. Dubois, MD, PhD, Chief Science Officer, National Pharmaceutical Council, 1717 Pennsylvania Ave., NW, Ste. 800, Washington, DC 20006. Tel.: 202.827.2079; Fax: 202.827.2100;E-mail: rdubois@npcnow.org.
3. Japsen B. Obamacare's accountable care approach reaches 1 in 10 in U.S. Forbes. November 26, 2012. Available at: http://www.forbes.com/sites/brucejapsen/2012/11/26/obamacares-accountable-care-approach-reaches-1-in10-in-u-s/. Accesssed October 16, 2013.

4. Gottlieb S. Accountable care organizations: the end of innovation in medicine? American Enterprise Institute. February 16, 2011. Available at: http:// www.aei.org/article/health/healthcare-reform/accountable-care-organizations-the-end-of-innovation-in-medicine/. Accessed October 16, 2013.

5. Kliff S. Get better! Your pharmacy's profits depend on it. The Washington Post. January 11, 2013. Available at: http://www.washingtonpost.com/blogs/ wonkblog/wp/2013/01/1l/get-better-your-pharmacys-profits-depend-on-it. Accessed October 16, 2013.

6. Hoyt RE, Bowling LS. Reducing readmissions for congestive heart failure. Am Fam Physician. 2001;63(8):1593-98.

7. Dubois RW, Feldman M, Martin J, Sanderson-Austin J, Westrich KD; Working Group on Optimizing Medication Therapy in Value-Based Healthcare. Role of pharmaceuticals in value-based healthcare: a framework for success. Am J Manag Care. 2012;18(7):e245-7. Available at: http://www.ajmc.com/publications/issue/2012/2012-7-vol18-n7/Role-ofPharmaceuticals-in-Value-Based-Healthcare-A-Framework-for-Success. Accessed December 5, 2013.

8. Jha AK, Burke MF, DesRoches C, et al. Progress toward meaningful use: hospitals' adoption of electronic health records. Am J Manag Care. 2011;17(12 Spec No):SP117-SP24. Available at: http://www.ajmc.com/publications/issue/2011/2011-12-vol17-SP/Progress-Toward-Meaningful-UseHospitalsu2019-Adoption-of-Electronic-Health-Records/. Accessed October 16, 2013.

9. Stenner SP, Chen Q, Johnson KB. Impact of generic substitution decision support on electronic prescribing behavior. J Am Med Inform Assoc. 2010;17(6):681-88. Available at: http://171.67.114.118/content/17/6/681.full Accessed October 16, 2013

10. Haas JS, Phillips KA, Gerstenberger EP, Seger AC. Potential savings from substituting generic drugs for brand-name drugs: medical expenditure panel survey, 1997-2000. Ann Intern Med. 2005;142(11):891-97. Available at: http:// annals.org/article.aspx?articleid=718428. Accessed October 16, 2013.

11. Chisholm-Burns MA, Kim Lee J, Spivey CA, et al. US pharmacists' effect as team members on patient care: systematic review and meta-analyses. Med Care. 2010;48(10):923-33.

12. Kohn LT, Corrigan JM, Donaldson MS, eds. To Err Is Human: Building a Safer Health System. Washington, DC: National Academies Press; 2000. Available at: http://www.nap.edu/catalog.php?record_id=9728. Accessed November 16, 2013

13. Centers for Medicare \& Medicaid Services. Readmissions reduction program. August 8, 2013. Available at: http://www.cms.gov/Medicare/MedicareFee-for-Service-Payment/AcuteInpatientPPS/Readmissions-ReductionProgram.html. Accessed December 2, 2013.

14. Barry MJ, Edgman-Levitan S. Shared decision making-the pinnacle of patient-centered care. N Engl J Med. 2012;366(9):78081. Available at: http:// www.nejm.org/doi/full/10.1056/NEJMp1109283. Accessed October 16, 2013.

15. Clancy CM. Patient engagement in health care. Health Serv Res. 2011;46(2):389-93. Available at: http://www.ncbi.nlm.nih.gov/pmc/articles/ PMC3064908/. Accessed October 16, 2013.

16. Berwick DM, Nolan TW, Whittington J. The triple aim: care, health, and cost. Health Aff (Millwood). 2008;27(3):759-69. Available at: http://content. healthaffairs.org/content/27/3/759.full. Accessed October 16, 2013. 


\section{APPENDIX ACO Self-Assessment Questionnaire}

\section{Demographic}

D1. What is your role in your organization?

D2. Which degrees do you possess (select all that apply)?

D3. How long have you been in your current role?

\section{Organizational}

O1. Do you own a health plan?

O2. What type of provider system is your ACO?

O3. How many physicians are in your system?

O4. How many hospitals are in your system?

O5. Are Long-Term Care and/or Specialty Offices part of the ACO structure?

O6. How many lives are included in your contract?

O7. What company/entity is your largest ACO contract with?

\section{Formularies}

F1. To what extent is a formulary in place to encourage use of generic drugs when appropriate?

F2. Is there a formal process for ensuring that formularies are synchronized across different sites of care (e.g., hospital, ambulatory)?

\section{Data Systems}

DS1. To what extent do physicians across the ACO have the ability to transmit prescriptions electronically to pharmacies via a computer or mobile device?

DS2. To what extent do care providers have the ability to view a patient's pharmacy and medical data within a single, centralized electronic data system?

DS3. To what extent are all of a patient's care providers notified when a medication is prescribed for a patient?

DS4. To what extent are all of a patient's care providers notified when a patient fills a prescription?

DS5. To what extent is an ACO-wide data system in place to identify potential drug-drug, drug-disease, and/or polypharmacy concerns?

DS6. To what extent are disease or chronic condition registries in place and capable of tracking patient prescriptions and drug use?

DS7. To what extent are existing systems able to alert providers of existing gaps in recommended preventative care (i.e., screenings, vaccinations, etc.)?

DS8. To what extent is the existing electronic system able to integrate or capture patient-reported outcomes, such as reasons for stopping a medication-plan or side effects experienced from medications?

DS9. To what extent are the necessary systems in place to determine if costs in one area (e.g., medications) offset costs or justify outcomes in other areas (e.g., emergency department, hospitalization)?

\section{Communication/Reporting}

CR1. When potential drug-drug, drug-disease, and/or polypharmacy concerns are identified, to what extent are protocols in place to ensure this information is shared with a patient's care team?

CR2. To what extent are patients educated about diagnostic and therapeutic alternatives and informed about the implications of such alternatives (e.g., complications, side effects) when determining the recommended medication care plan?

CR3. To what extent are clinical pharmacists involved in direct patient care? For example, providing medication management counseling, guidance for home infusion therapy, medication management, or pharmacy-based clinical services?

CR4. When there are condition-specific incentives to achieve economic savings (e.g., substitute lower cost therapies for higher cost ones), to what extent are there corresponding evidence-based quality metrics and incentives to ensure appropriate medication use?

\section{Standard Care Plan}

SCP1. To what extent does each clinical team across the system have standard protocols in place to avoid duplicate medications, polypharmacy, and adverse impacts to medications?

SCP2. To what extent do visit summaries include a list of medications, potential adverse reactions, and clear directions for taking the prescribed drug(s)?

\section{ACO Contract}

ACO1. To what extent is the ACO financially responsible for oral/pharmacy-based (e.g., Part D) medications? 